\title{
Endoglin and activin receptor-like-kinase 1 are co-expressed in the distal vessels of the lung: implications for two familial vascular dysplasias, $\mathrm{HHT}$ and PAH
}

\author{
Marwa Mahmoud', Gillian M Borthwick', Alison A Hislop² and Helen M Arthur ${ }^{1}$
}

Arteriovenous malformations (AVMs) are direct connections between arteries and veins associated with loss of the intervening capillary bed. In the lungs, pulmonary AVMs can result in right to left shunts and severe cyanosis and dyspnoea. However, the cellular and molecular mechanisms underlying AVM formation are poorly understood. One important clue comes from the fact that pulmonary AVMs frequently occur in the familial disease hereditary haemorrhagic telangiectasia (HHT), which is associated with mutations in one of two receptors involved in transforming growth factor- $\beta$ family signalling, either endoglin (ENG) or activin receptor-like kinase 1 (ACVRL1, also known as ALK1). To elucidate the potential link between ENG or ACVRL1 deficiency and AVM formation in HHT, we performed a comprehensive study of Acvrl1 and Eng expression in wild-type and Eng-deficient (Eng $+/-$ ) mouse lungs using a combination of immunohistochemistry and RT-PCR from laser-microdissected arteries, veins and capillaries. We found that Eng and Acvrl1 have distinct expression profiles in the pulmonary vasculature and are only co-expressed in the distal (pre-capillary) arteries, distal veins and capillaries, consistent with the tendency for pulmonary AVMs to form in the distal pulmonary vessels in HHT. Downstream pSmad1/5/8 activity was found in the distal arteries and was specifically reduced in Eng $+/-$ mice, consistent with previous in vitro data showing that Eng promotes Acvrl1-mediated Smad1/5/8 phosphorylation. Eng was more widely expressed than Acvrl1 in the lungs, as Eng alone was found in pulmonary veins, potentially explaining the increased frequency of AVMs in HHT1 patients. Furthermore, the association of ACVRL1 mutations with a second vascular disease, familial pulmonary artery hypertension, underlines the importance of ACVRL1 expression in the distal arteries that are affected in this disorder.

Laboratory Investigation (2009) 89, 15-25; doi:10.1038/labinvest.2008.112; published online 17 November 2008

KEYWORDS: familial pulmonary artery hypertension; hereditary haemorrhagic telangiectasia; pre-capillary arterioles; transforming growth factor- $\beta$; arteriovenous malformation

Activin receptor-like-kinase 1 (ACVRL1, also known as ALK1) and endoglin (ENG) are receptors for ligands of the transforming growth factor- $\beta$ (TGF- $\beta$ ) family and are expressed on the surface of vascular endothelial cells. Mutations in the ENG and ACVRL1 genes are associated with two different familial vascular dysplasias, hereditary haemorrhagic telangiectasia (HHT) and, to a lesser degree, with familial pulmonary artery hypertension (fPAH). ${ }^{1}$ HHT is a multisystemic vascular dysplasia characterized by dilated vessels or telangiectases on mucocutaneous surfaces and arteriovenous malformations (AVMs) in the lung, liver and brain. ${ }^{2}$ AVMs are direct connections between arteries and veins associated with vessel dilatation and loss of the intervening capillary bed. In the lungs, pulmonary AVMs (PAVMs) result in right to left shunting of blood that can lead to severe cyanosis and dyspnoea. ${ }^{3}$ Moreover, $\mathrm{fPAH}$ is characterized by partial occlusion of the distal pulmonary arterioles because of local endothelial and vascular smooth muscle cell proliferation. ${ }^{4}$ Familial PAH is associated with mutations in the BMP type II receptor, and more rarely with ACVRL1 or ENG mutations. ${ }^{5-7}$ Both diseases cause major mortality and morbidity, but little is known about the molecular and cellular mechanisms un-

\footnotetext{
${ }^{1}$ Institute of Human Genetics, Centre for Life, Newcastle University, Newcastle upon Tyne, UK and ${ }^{2}$ Institute of Child Health, London, UK Correspondence: Dr HM Arthur, PhD, Institute of Human Genetics, Centre for Life, Newcastle University, Newcastle upon Tyne NE1 3BZ, UK. E-mail: helen.arthur@ncl.ac.uk
} 
derlying the formation of these two quite different vascular dysplasias.

The incidence of HHT in the general population has been reported to be as high as 1 in $5000 .^{8-10}$ It is inherited as an autosomal dominant disorder, with varying penetrance and clinical severity even among members of the same family. To date, five genetically distinct, but phenotypically similar forms of HHT have been identified. HHT type 1 is caused by mutations in the ENG gene, whereas HHT type II is caused by mutations in the ACVRL1 gene. ${ }^{11,12}$ The causative genes for HHT types III and IV have not yet been identified, ${ }^{13,14}$ while a combined syndrome of juvenile polyposis and HHT is associated with mutations in the SMAD4 gene. ${ }^{15}$ All the known genes implicated in HHT and PPAH encode proteins belonging to the TGF- $\beta$ family signalling pathway, and mutations in ACVRL1 and ENG genes are the only ones to date that have been found in both vascular dysplasias.

Endoglin is an auxiliary receptor that promotes signalling through ACVRL1 in response to different ligands of the TGF- $\beta$ family, including TGF- $\beta 1$ and BMP9. ${ }^{16-18}$ Therefore, it is likely that the abnormal vessels in HHT and PAH develop because of perturbed TGF- $\beta$ family signalling in vascular endothelial cells during vascular development and/or adult life. However, the aberrant molecular and cellular responses that underlie these vascular dysplasias are poorly understood.

The first stage in the development of an AVM in HHT is thought to be a focal dilatation and elongation of a postcapillary venule, followed by the establishment of a direct connection with an enlarging arteriole, and associated loss of the intervening capillary bed. ${ }^{19}$ Clinical symptoms often begin in puberty with bleeding episodes from small AVMs (telangiectases) in the nasal mucosa, whereas further manifestations of the disease continue to develop in later life. PAVMs are often multiple and tend to occur in the lower lobes that might be because of this being the major region of pulmonary blood flow in humans. ${ }^{3}$ They usually occur sub-pleurally indicating a distal, rather than a proximal, location in the vasculature. ${ }^{20}$ In addition, PAVMs occur at higher frequency $(30 \%)$ in HHT1 compared with HHT2 patients, pointing to a more critical role for ENG than for ACVRL1 in maintaining normal pulmonary vasculature. ${ }^{21}$

In $\mathrm{fPAH}$, the first stage of the disease appears to be proliferation of endothelial and smooth muscle cells to partially occlude the distal arteries, followed by the formation of secondary multiple vascular channels nearby to form a complex plexiform lesion. ${ }^{4}$ However, despite the involvement of ACVRL1 and ENG in both these pulmonary vascular dysplasias, current knowledge of expression of these genes in the pulmonary vasculature is limited. Previous work has shown that Acvrll is expressed primarily in arterial endothelial cells during development, ${ }^{22-24}$ and in adult life remains highly expressed in lung vasculature. ${ }^{25}$ In the developing embryo, Eng is more widely expressed throughout the vascular bed, ${ }^{26}$ but is downregulated in adult life, with the notable exception of pulmonary vessels. ENG expression can also respond locally to a range of stimuli and is upregulated during wound healing, inflammation and tumour angiogenesis. ${ }^{27,28} \mathrm{~A}$ recent study has shown that TGF- $\beta$ receptors are dynamically regulated during early lung development, ${ }^{29}$ but a detailed spatial study of ENG and ACVRL1 expression in different regions of the pulmonary vasculature has not yet been carried out.

To elucidate the potential link between ENG or ACVRL1 deficiency and their association with vascular dysplasia in fPAH and HHT, we performed a comprehensive study of Acvrll and Eng expression throughout the murine pulmonary vascular tree in adult and early postnatal life. We compared wild-type and Eng $+/$ - mice using a combination of immunohistochemistry on tissues sections and RT-PCR from laser-microdissected blood vessels. We show distinct expression profiles for these genes in the pulmonary vasculature, pointing to a role for these genes in the distal vessels, and consistent with the involvement of these regions in HHT and $\mathrm{PPAH}$.

\section{MATERIALS AND METHODS \\ Mice}

The Eng $+/-$ mice, used in this study have been described previously, were maintained as a heterozygous breeding colony in an NIH background (backcrossed for 16 generations) and are asymptomatic for HHT-like lesions. ${ }^{30}$ At least four matched mutant and wild-type mice from a minimum of three litters were used for protein and RNA expression analysis. Lungs from adult male mice (age approximately 12 weeks) and early postnatal mice (5-day-old) were prepared by gently flushing the vasculature with PBS via the right ventricle (a small cut in the left atrium allowed outflow of perfusate). Lungs were gently inflated by injecting a 50:50 mixture of Tissue-TEK OCT and PBS through the trachea, snap frozen in liquid-nitrogen-cooled isopentane and orientated for cutting coronal sections. All animal procedures were approved by local ethics committee.

\section{Immunostaining}

Immunohistochemistry was performed on cryosections fixed in acetone, blocked with diluted goat or rabbit serum and incubated with the appropriate primary antibody overnight at $4{ }^{\circ} \mathrm{C}$. Non-mouse primary antibodies were endothelial nitric oxide synthase (eNOS; BD Transduction Laboratories), ENG clone MJ7/18 (eBioscience) and CD31 clone Mec13.3 (BD Biosciences); cyclooxygenase-2 (Cox-2; Santa Cruz Biotechnology), Alk-1 (R\&D Systems), anti-pSmad2 and anti-pSmad1/5/8 (NEB). The biotinylated secondary antibody was goat anti-rabbit, rabbit anti-rat or rabbit anti-goat (Vector), diluted in goat or rabbit serum and detected using $\mathrm{ABC}$ reagent (Vector) and $\mathrm{DAB}$ solution. For mouse primary antibodies, $\alpha$-smooth muscle actin, $\alpha$-actinin (Sigma) and HistoMouse kit (Invitrogen), were used. Sections were counterstained with Mayer's Haematoxylin. For pSmad immunostaining, tissue was fixed with $0.2 \%$ PFA overnight at 
$4^{\circ} \mathrm{C}$ and citrate antigen retrieval was used to expose the epitopes. At least three biological replicates were analysed at each age to confirm pattern of immunostaining. Fluoresceinlabelled GSL I-isolectin $\mathrm{B}_{4}$ (Vector) was used to stain endothelial cells.

\section{Laser Microdissection and RT-PCR}

Cryosections on PET (polyethylene terephthalate) membrane metal frame slides (Leica) were rapidly counterstained with Mayers Haematoxylin and dehydrated in a graded alcohol series (using RNase free water) before LMD with the Leica AS System. Adjacent sections on poly-L-lysine microscope slides were stained for CD31, Eng and Acvrl1 to identify the vessels of interest. Target cells from capillaries, veins, arteries and bronchioles were isolated by laser microdissection and collected into lysis buffer with $\beta$-mercaptoethanol (RNeasy Micro Kit, Qiagen) before RT-PCR using SuperScript III One-Step System with Platinum Taq DNA Polymerase (Invitrogen). The following forward $(\mathrm{F})$ and reverse $(\mathrm{R})$ primer pairs (all written $5^{\prime}-3^{\prime}$ ) were used: $\beta$-actin, F tgaaccctaaggccaaccgtg and R gctcatagctcttctccaggg; Acvrll, $\mathrm{F}$ gaccttggggagcttcaga and R tgcagaaggatctatagcagca; Pecam1, F aagcggtcgtgaatgacac and $\mathrm{R}$ tttggctgcaactattaaggtg; ENG, $\mathrm{F}$ caatgccagcattgtcacctcc and $\mathrm{R}$ agaggctgtccatgtcgatgca. PCR products were separated by electrophoresis in agarose gels and stained with ethidium bromide before capturing images using a Typhoon Trio scanner (GE Healthcare). Band intensities were quantified using ImageQuant TL software (GE Healthcare).

\section{Western Blotting}

Lung proteins were extracted from whole lungs of matched adult ( $\geq 8$ weeks) male mutant and wild-type mice by homogenization in lysis buffer $(10 \mathrm{mmol} / \mathrm{l}$ Tris- $\mathrm{HCl}, \mathrm{pH} 7.4$, $1 \%$ Triton X-100, protease inhibitors (Sigma) and phosphatase inhibitors (Roche)) and solubilized in SDS sample buffer before separation on a $10 \%$ SDS polyacrylamide gel. Proteins were transferred to Hybond membrane and blocked in 5\% milk or $2 \%$ gelatin. Primary antibodies were as above except for anti-eNOS (C-20) rabbit polyclonal antibody-HRP conjugate (Santa Cruz Biotechnology), monoclonal anti- $\beta$-tubulin clone TUB2.1 (Sigma), Smad 1 (A-4) mouse monoclonal IgG (Santa Cruz Biotechnology) and Smad 2 (L16D3; NEB). All secondary antibodies were conjugated to HRP and used at a 1:10 000 dilution. HRP was detected using SuperSignal West Pico Chemiluminescent Substrate (Pierce Biotechnology), visualized after exposure to X-ray film and band intensities were quantified using ImageQuant TL software (GE Healthcare).

\section{Statistical Analysis}

Results are represented as mean \pm standard deviation. Statistical significance was determined using the unpaired $t$-test and $P<0.05$ was considered statistically significant.

\section{RESULTS}

In the murine lung, pulmonary veins are easily recognized by the distinctive myocardialization of the vascular wall ${ }^{31,32}$ (Figure $1 \mathrm{a}$ and $\mathrm{b}$ ). In contrast, pulmonary arteries lack myocardial cells and are found in close proximity to bronchioles in the bronchiovascular bundle (Figure 1c). Eng expression was found throughout the endothelium of the pulmonary veins and venules in adult wild-type mice, but was not detected in the majority of pulmonary artery endothelium. Positive Eng staining was found in the pulmonary veins, in the artery valve leaflets and in the neighbouring arterial endothelium (Figure 1d). However, Eng was downregulated as the pulmonary artery divides into the left and right arteries just before entering the lung parenchyma (Figure 1d and e). Eng expression remained at undetectable levels in the pulmonary artery until the distal arterioles where it was clearly visible (Figure if and g). In contrast, Acvrll was not detected in pulmonary veins or pulmonary arteries except in the endothelium of distal venules and arterioles (Figure 2). Both Eng and Acvrll are strongly expressed in the alveolar capillaries. Therefore, in the lung, Acvrl1 expression only overlaps with Eng in distal arterioles, distal venules and capillaries.

To confirm pulmonary expression patterns of Acvrl1 and Eng using an independent approach, we isolated RNA from murine pulmonary arteries, veins and capillaries by means of laser microdissection. Acvrl1 and Eng expression at the RNA level was compared against a housekeeping gene ( $\beta$-actin) using RT-PCR. Figure 3 shows that Eng RNA was found in veins and capillaries, but not arteries, while Acvrl1 RNA was seen only in capillaries and is consistent with the protein pattern seen with immunohistochemistry. We were unable to test expression in the distal arteries and distal veins because of their limited size and frequency.

To define the transition point in the distal vasculature at which Acvrll and Eng expression occurs, we mapped the branch points of the arteries and bronchioles in the lungs of wild-type mice in a similar way to that previously carried out in human lungs. ${ }^{33}$ Positive staining for both receptors starts at the same point at approximately the fourth branch point of the bronchiolar tree (Figure 4). This could be recognized in individual sections because these small bronchioles had decreased smooth muscle mass and 'patchy' smooth muscle actin staining in their walls (Figure 4b), and which we defined as small bronchioles. These contrasted with the larger bronchioles that had a thicker and less interrupted smooth muscle layer in their walls (Figure 4a). As expected, in lungs from heterozygous Eng $+1-$ mice, the level of Eng was reduced to approximately $50 \%$ that of wild-type mice, but showed a similar spatial pattern of expression. However, careful observation revealed that the transition point in the distal arterioles had moved toward the capillaries. Importantly, there was no observed effect of Eng reduction on the level or pattern of Acvrll expression (Figure 4i and $j$ ). This meant that in Eng $+/-$ mice, Acvrll was expressed in 

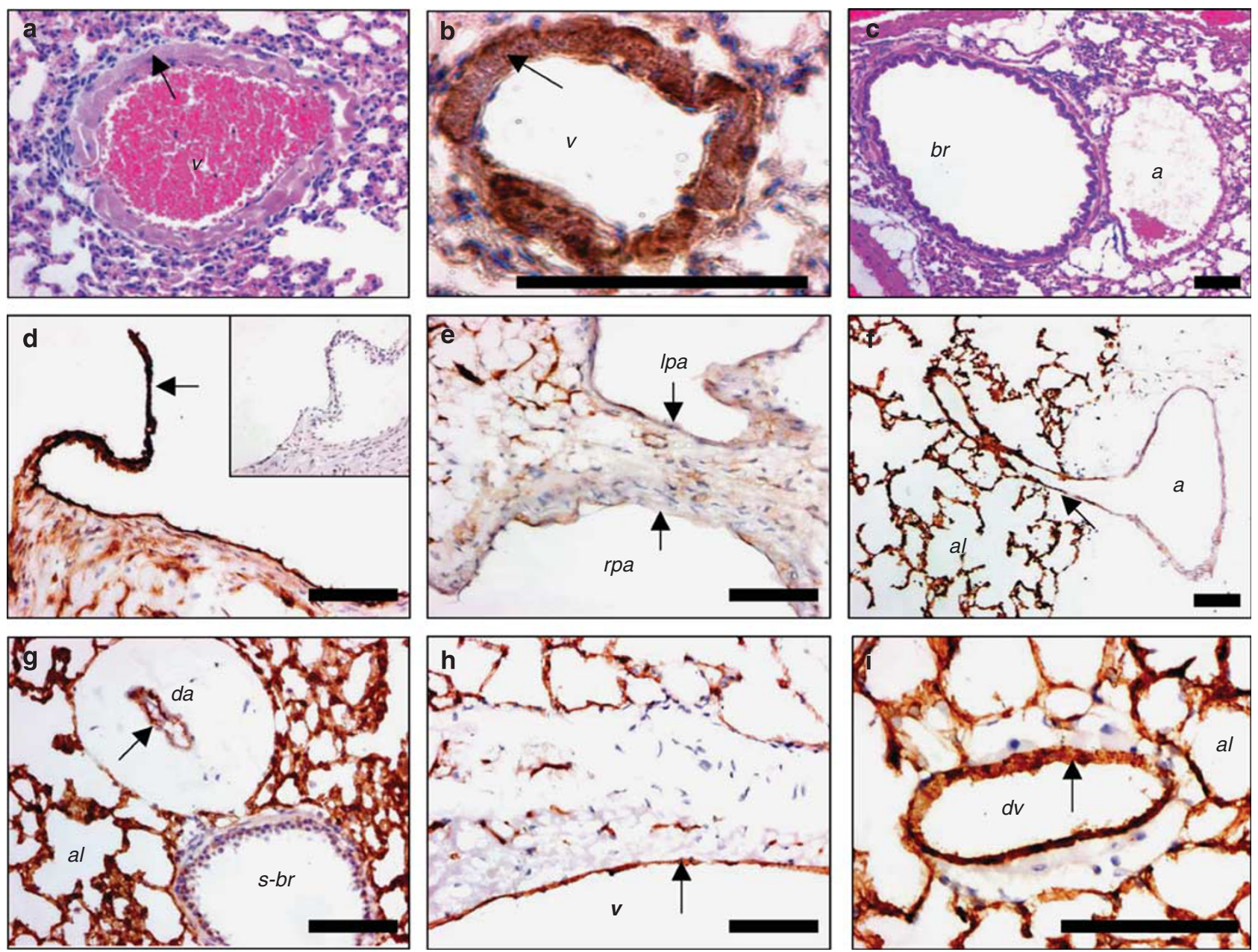

Figure 1 Endoglin is expressed in the veins, distal arteries and capillaries of the pulmonary vasculature. Pulmonary veins are supported by distinctive myocardial muscle cells (arrows) that are recognizable in H\&E-stained sections (a), and confirmed by cardiac $\alpha$-actinin staining (b). Pulmonary arteries are recognized by their position within a bronchiovascular bundle in H\&E-stained sections (c). Endoglin is expressed in the pulmonary artery valve leaflets (arrow) and surrounding endothelium (d); but expression is lost (arrows) as the pulmonary artery divides into the left and right pulmonary arteries (e). (Inset in $\mathbf{d}$ shows an example of negative staining in no primary antibody controls). Expression remains low in arteries within the lung parenchyma, until the distal arteries where there is a transition to positive expression (arrows; $\mathbf{f}, \mathbf{g}$ ). Endoglin is expressed throughout the venous (arrows) and capillary endothelium (h, i). Abbreviations: a, artery; al, alveolus; br, bronchiole; da, distal artery; dv, distal vein; Ipa, left pulmonary artery; rpa, right pulmonary artery; s-br, small bronchiole; $v$, vein. Scale bars: $100 \mu \mathrm{m}$.

small regions of the distal arteries where Eng was below detectable limits (orange regions in Figure 4b).

We next examined whether the expression pattern for Eng and Acvrll was established earlier in postnatal life. At day 5, the lung is rapidly growing, but we found that the spatial expression pattern of Eng and Acvrl1 remains essentially unchanged between postnatal day 5 and adult (not shown).

Endoglin enhances the ability of Acvrll to promote TGF- $\beta$ family signalling through activation of the BMP responsive Smads (Smads1, 5 and 8). ${ }^{34}$ Smad activation can be readily seen in vivo using antibodies that specifically recognize the phosphorylated (p) Smad forms. At postnatal day 5, pSmad1/ $5 / 8$ activity is seen throughout vasculature (not shown), but becomes restricted to distal arterioles, venules and capillaries (areas of Eng and Acvrll colocalization) in adult lungs (Figure 5). This is consistent with high levels of BMP signalling in the rapidly growing postnatal lung, but a more restricted pattern of BMP signalling in adult lung. The pattern of pSmad 1/5/8 expression in adult lungs is consistent with activation of the Acvrll signalling pathway in the distal arterioles and venules, although activity of other BMP responsive receptors cannot be ruled out.

To investigate whether reduced Eng levels affected signalling in vivo, we examined $\mathrm{pSmad} 1 / 5 / 8$ in lung sections from wild-type and Eng $+/-$ mice using immunohistochemistry and we found reduced pSmad1/5/8 activity in distal artery endothelial cells in the mutants compared to the controls (Figure $5 \mathrm{~h}$ and $\mathrm{g}$ ). We then compared pSmad1/5/8 expression in wild-type and Eng + /adult lung extracts using western blots and found that pSmad1/5/8 levels were significantly reduced in Eng $+/-$ lungs (Figure $5 \mathrm{i}$ and $\mathrm{j}$ ). 

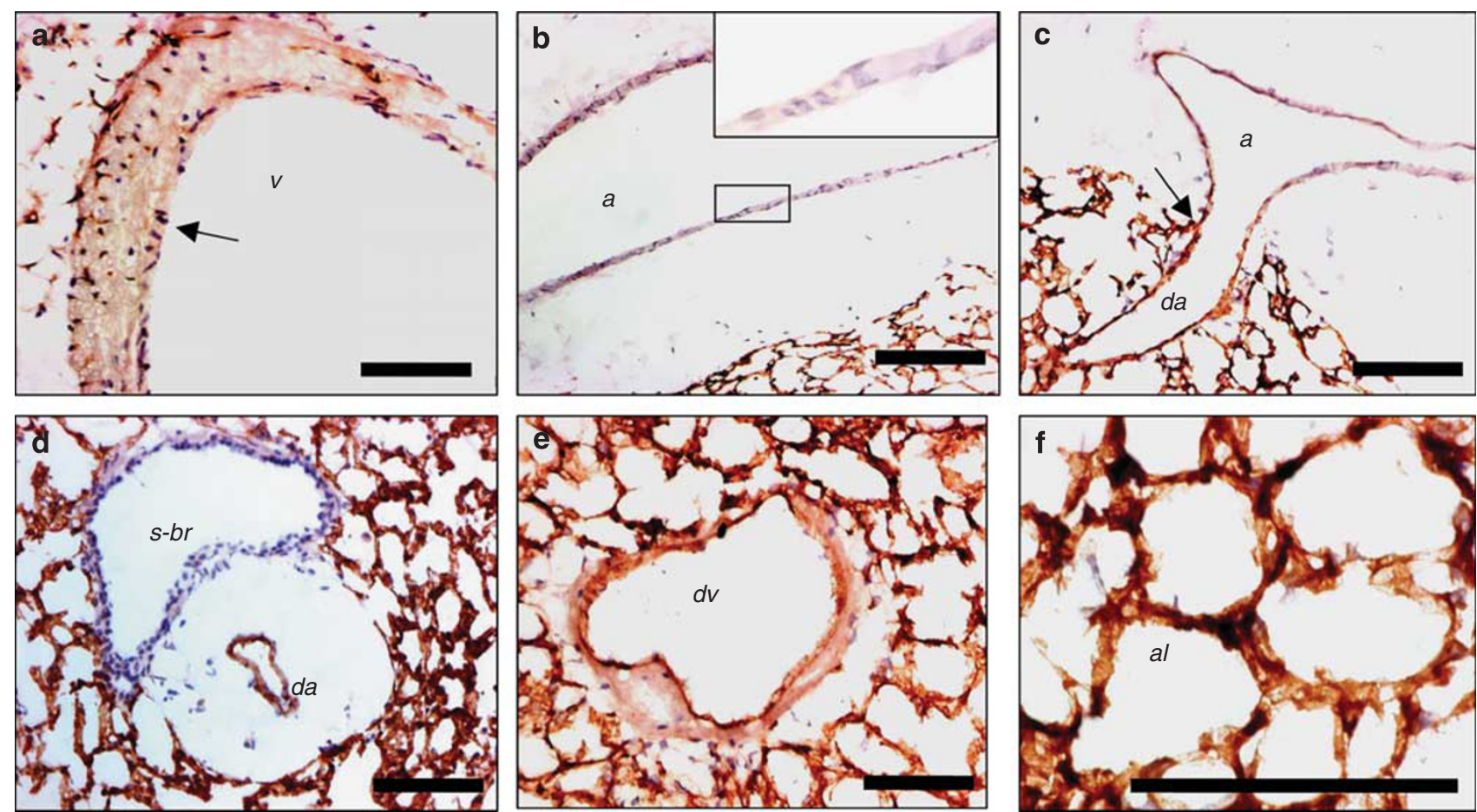

Figure 2 Acvrl1 is expressed in the distal veins, distal arteries and capillaries of the pulmonary vasculature. Acvrl1 is not expressed in major pulmonary arteries and veins $(\mathbf{a}, \mathbf{b})$, but is present in distal arteries and distal veins (c-e). Inset in (b) shows magnified view. The transition point between nonexpressing and Acvrl1-expressing arterial endothelium (arrow, c) is at a similar point to that for endoglin expression. Acvrl1 is strongly expressed in the capillaries (f). For abbreviations see legend of Figure 1. Scale bars: $100 \mu \mathrm{m}$.

a

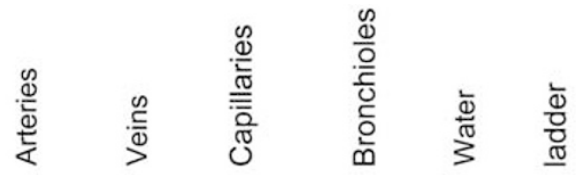

Endoglin $\quad-10 \times-1$

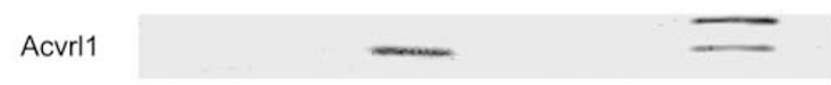

Pecam-1

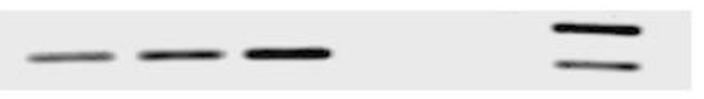

$\beta$-actin

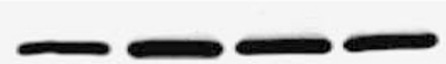

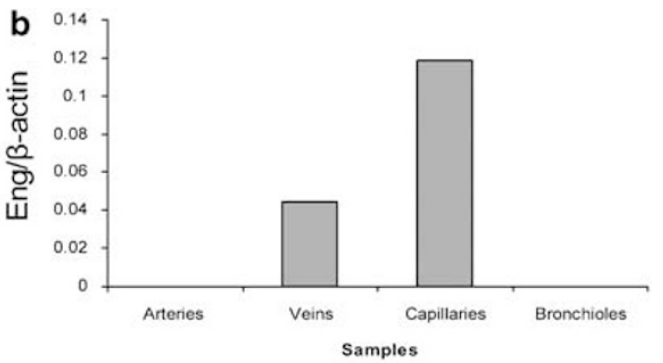

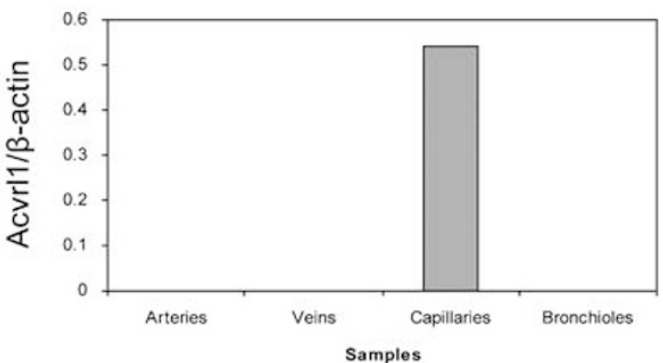

Figure 3 Expression of Acvrl1 and endoglin RNA using laser microdissection and RT-PCR. RNA was isolated from pulmonary arteries, veins, capillary regions and bronchioles and endoglin, Acvrl1, Pecam-1 and $\beta$-actin expression were detected by RT-PCR products, visualized on ethidium-stained gels. A representative gel from three independent experiments is shown (a) and the relative intensity of the endoglin and Acvrl1 PCR products is illustrated (b). Endoglin and Acvrl1 RNA are not detected in proximal arteries or the bronchioles, only endoglin is detected in the proximal veins, while both receptors are seen in capillaries.

We have previously observed that Eng activity in endothelial cells had a downstream paracrine effect on TGF- $\beta$ signalling to neighbouring cells, which could be detected by a reduced pSmad2 response. ${ }^{35}$ In adult and postnatal lungs, $\mathrm{pSmad} 2$ is present throughout all endothelial cells and the supporting smooth muscle cells (Figure 6), suggesting Alk5 
a
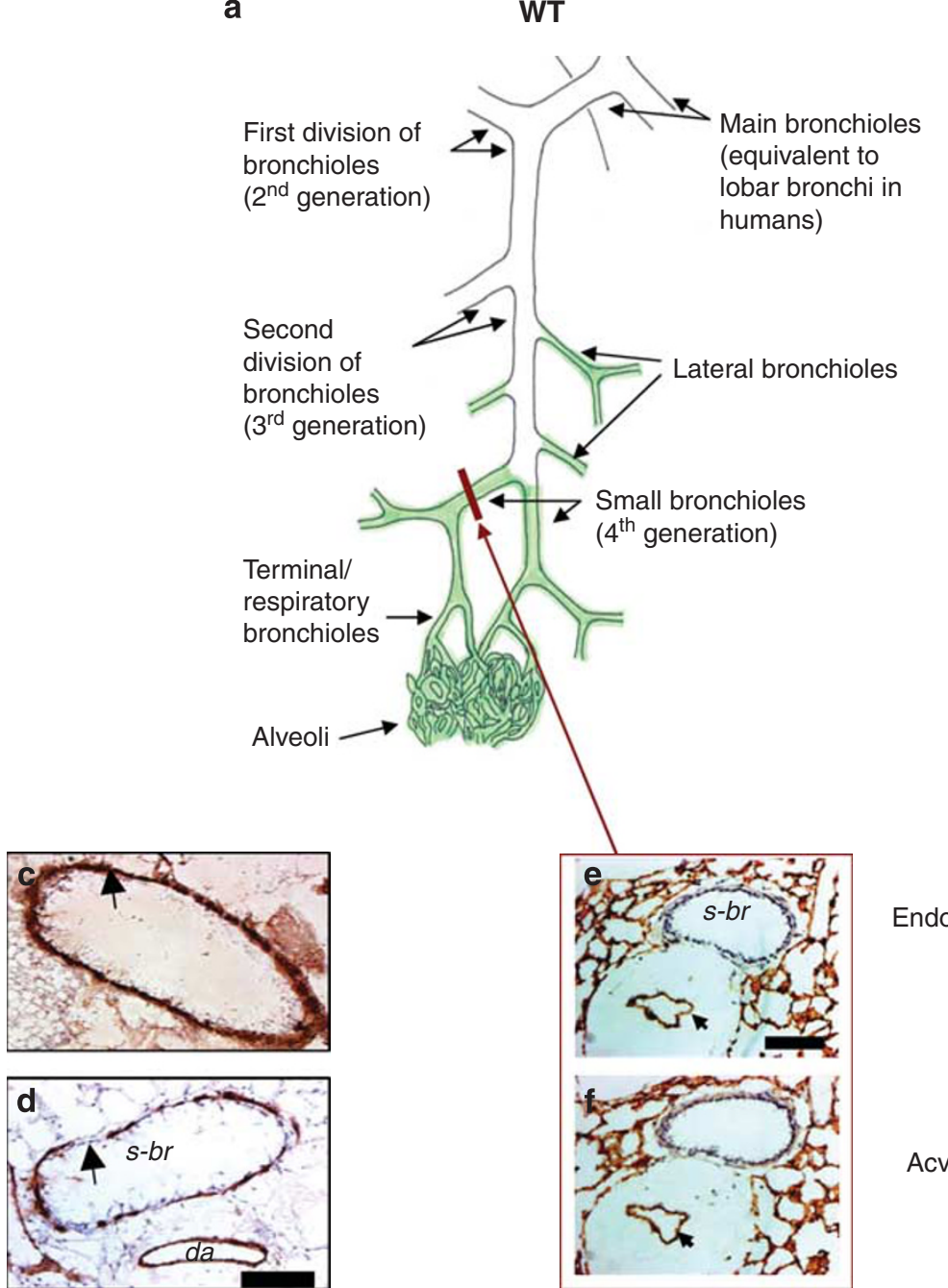

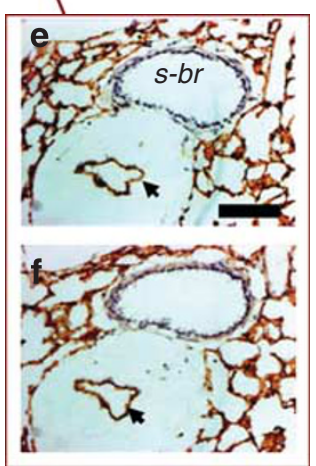

Eng +/-

b

\section{Eng $+1-$}

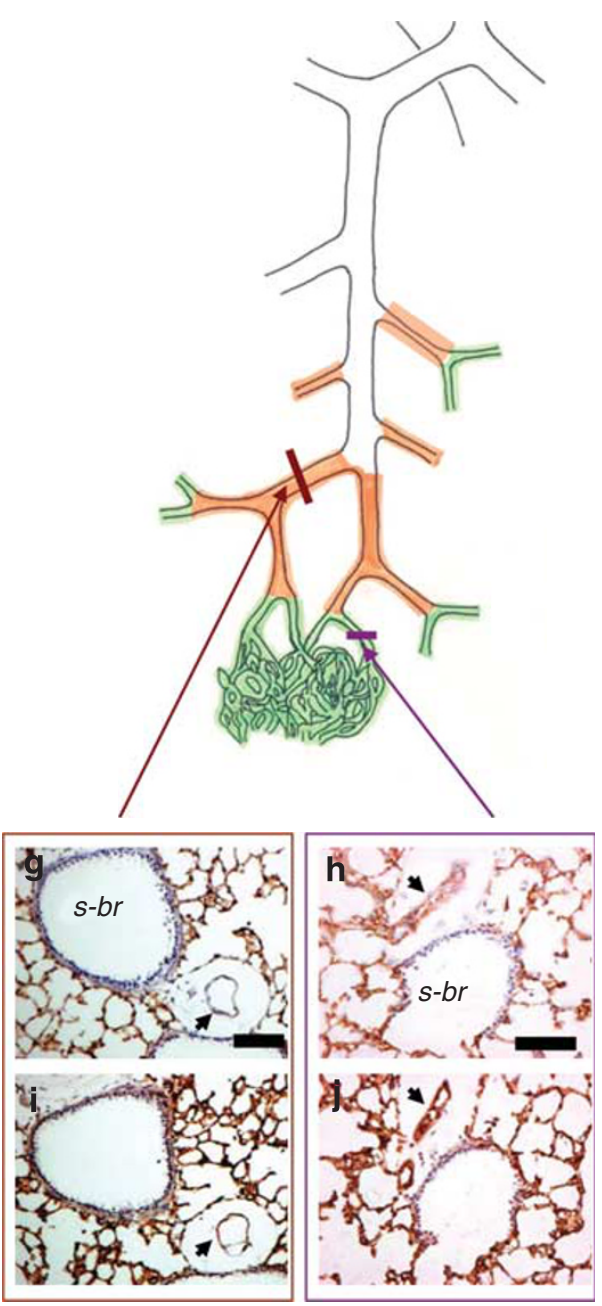

Figure 4 A cartoon summarizing differences in endoglin and Acvrl1 expression in the pulmonary arterial vasculature of wild-type (a) and heterozygous Eng $+/-(\mathbf{b})$ mice. Arteries are mapped according to where they run alongside the bronchial tree. In WT mice, Acvrl1 and endoglin expression are not detected in endothelial cells lining the proximal arteries and are only detected at the level of the distal arteries alongside small bronchioles (a, green). Proximal arteries ran alongside bronchioles with uninterrupted $\alpha$-smooth muscle actin staining in their walls (c), arrow) and are white in the cartoon (a), whereas distal arteries corresponding to the green regions (a) were adjacent to small bronchioles with patchy $\alpha$-smooth muscle actin staining (d, arrow). Loss of a single Eng allele (in the Eng $+/$ - mice) leads to an overall reduction in endoglin expression (compare $\mathbf{e}$ with $\mathbf{g}$ and $\mathbf{h}$ ), but no change in Acvrl1 expression (compare $\mathbf{f}$ with $\mathbf{i}$ and $\mathbf{j}$ ). There is also loss of detectable endoglin expression in the distal arteries (orange, $\mathbf{b}$; arrow, $\mathbf{g}$ ), leaving only small regions of endoglin and Acvrl1 co-expression in the extremely distal arteries (green, $\mathbf{b}$ ) shown in serial sections (arrows in $\mathbf{h}$ and $\mathbf{j}$ ). Note that distal arteries expressing Acvrl1 in the absence of detectable endoglin are seen only in the Eng $+/$ - mice (orange) shown in serial sections (arrows in $\mathbf{g}$ and $\mathbf{i}$ ). For abbreviations see legend of Figure 1.

(or Activin) signalling is constitutively active. The wideexpression pattern of $\mathrm{pSmad} 2$ in the lung suggests there is no particular association of this activity with Eng or Acvrll expression. However, we were interested to know whether pSmad2 activity in cells adjacent to endothelial cells (presumed smooth muscle cells) in the distal arteries was affected in Eng $+/-$ mice. Consistent with previous data, there appeared to be a reduction in pSmad2 activity in both endothelial cells and smooth muscle cells in the distal arteries as judged by immunofluorescence, and in western blots of total lung extract (Figure 6). However, pSmad2 levels were not significantly reduced in the total lung extracts
(Figure 6d). Extensive epithelial pSmad2 activity may mask any reduction in vascular pSmad levels in Eng $+/$ - total lung extracts and further work is therefore required to establish the extent of vascular-specific changes in pSmad2 activity.

It has been hypothesized that disruption of vascular tone leading to lack of flow regulation contributes to the development of AVMs. ${ }^{36}$ The expression of Cox- 2 and eNOS, two proteins involved in regulating vascular tone, have been found to be affected in Eng +1 - heterozygous mice and also in cultures of blood outgrowth endothelial cells from HHT patients, suggesting a link between endoglin expression levels and these vasoregulators. ${ }^{37-39}$ We therefore examined the 

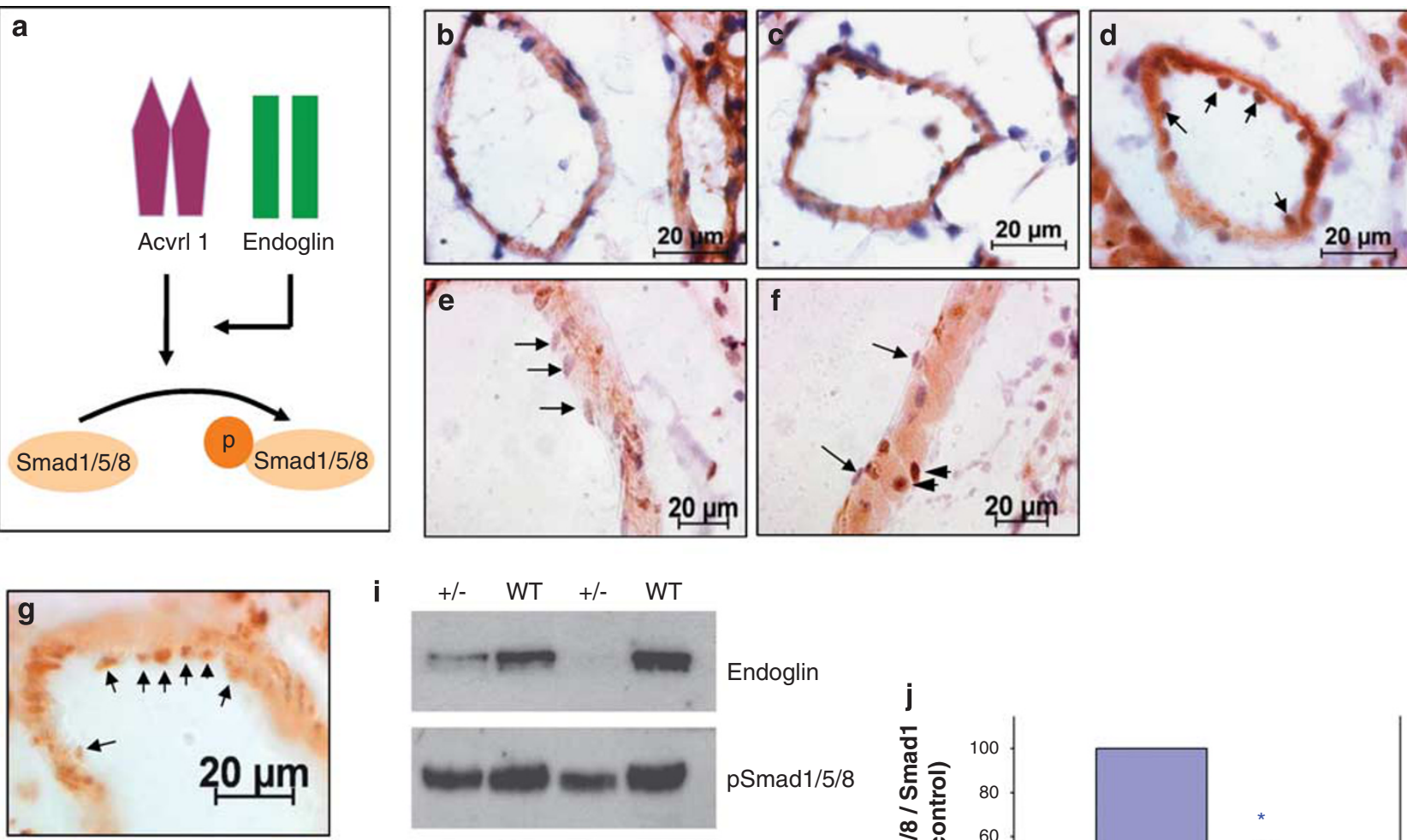

\section{i}
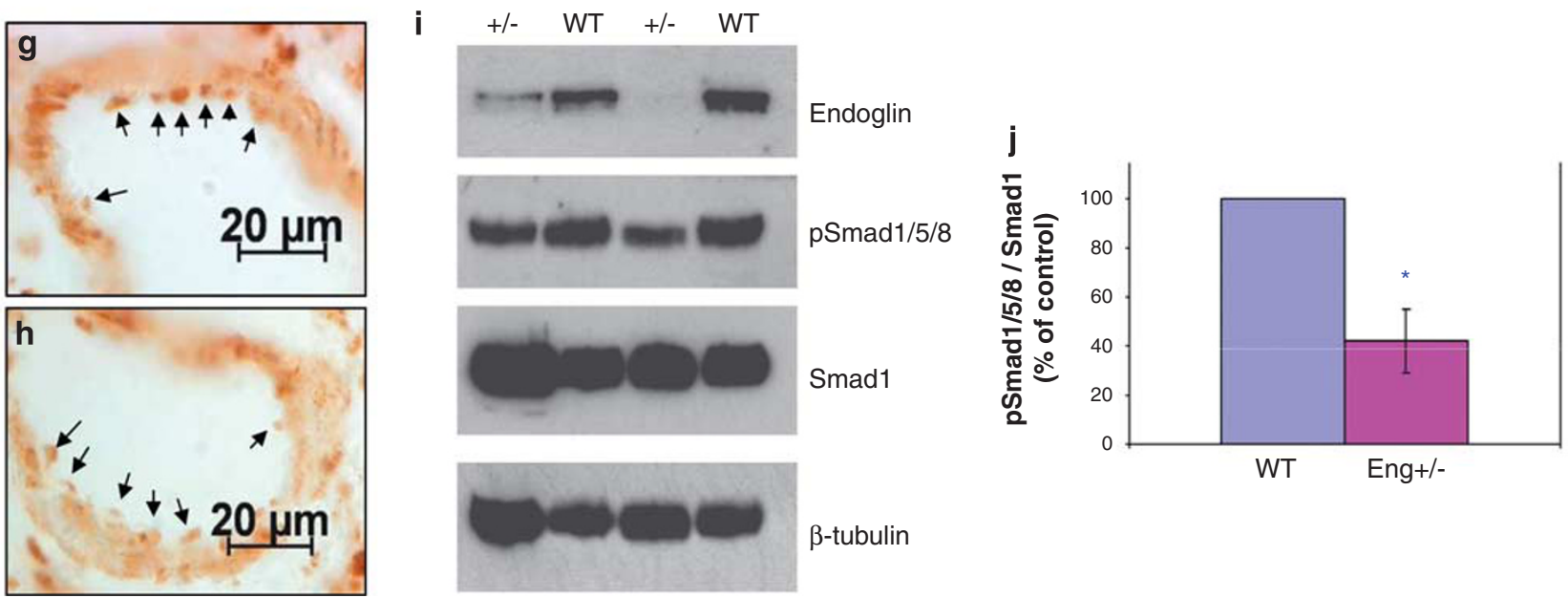

Figure 5 pSmad1/5/8 activity is associated with regions of endoglin and Acvrl1 co-expression and is reduced in Eng $+/-$ lungs. Diagram illustrating that endoglin promotes Acvrl1 phosphorylation of Smad1/5/8 (a). Serial sections of a distal artery showing co-expression of endoglin (b), Acvrl1 (c) and pSmad1/ $5 / 8$ (d) in endothelial cells. In adult lung, there was no pSmad1/5/8 detected in endothelium (arrows) of main arteries (e) or veins (f), although positive staining can be seen in some of the cardiac muscle cells (arrowheads, f). pSmad1/5/8 activity in distal arteries of wild-type lungs (g) appeared greater than that of Eng $+/$ - lungs (h) and a western blot of total lung protein extract also showed reduced pSmad1/5/8 activity in Eng $+/-$ lungs. Densitometric analysis of the western blots of total protein from lungs of four wild-type and four Eng $+/-$ mice $(\mathbf{i}, \mathbf{j})$ confirmed pSmad $1 / 5 / 8$ activity was significantly reduced in Eng $+/$ - lungs $\left({ }^{*} P=0.0013\right)$.

expression of eNOS and Cox-2 in the vasculature in adult wild-type and Eng $+/$ - mice to determine whether sites of expression of these enzymes would correlate with Eng and Acvrll expression. eNOS was present in the pulmonary arterial endothelium, in both the proximal and distal branches and also in the capillaries (Figure 7). However, no eNOS expression was detected in the endothelium of the pulmonary veins that would correspond with Eng expression, even though strong eNOS expression occurred in the supporting layer of myocardium (Figure $7 \mathrm{~b}$ and $\mathrm{d}$ ), as previously reported. ${ }^{40}$ In addition, we were surprised to find that Cox-2 displayed a reciprocal expression pattern to Eng in the pulmonary arteries. Cox-2 expression was detected in the proximal pulmonary arteries but was absent in the distal pulmonary arteries where Eng expression was clearly visible (Figure 7e-h). Moreover, Cox-2 expression was detected throughout the pulmonary veins and capillaries (not shown). Using immunohistochemistry, we were unable to detect a difference in expression levels of either Cox-2 or eNOS in the pulmonary vasculature of the Eng $+/$ - mice compared with wild type, but densitometric analysis of western blots did indicate a small reduction in both Cox-2 and eNOS expression in Eng $+/$ - lungs (Figure 7).

\section{DISCUSSION}

We have shown that the expression of Eng and Acvrll in the pulmonary vascular endothelium is non-uniform. High levels of co-expression are seen in the distal vessels of mouse lung. We attempted to verify this pattern in human using ex-transplant human lung tissue. However, all samples obtained had some degree of inflammatory infiltrate, which is known to affect ENG expression. ${ }^{27}$ Therefore, we cannot 

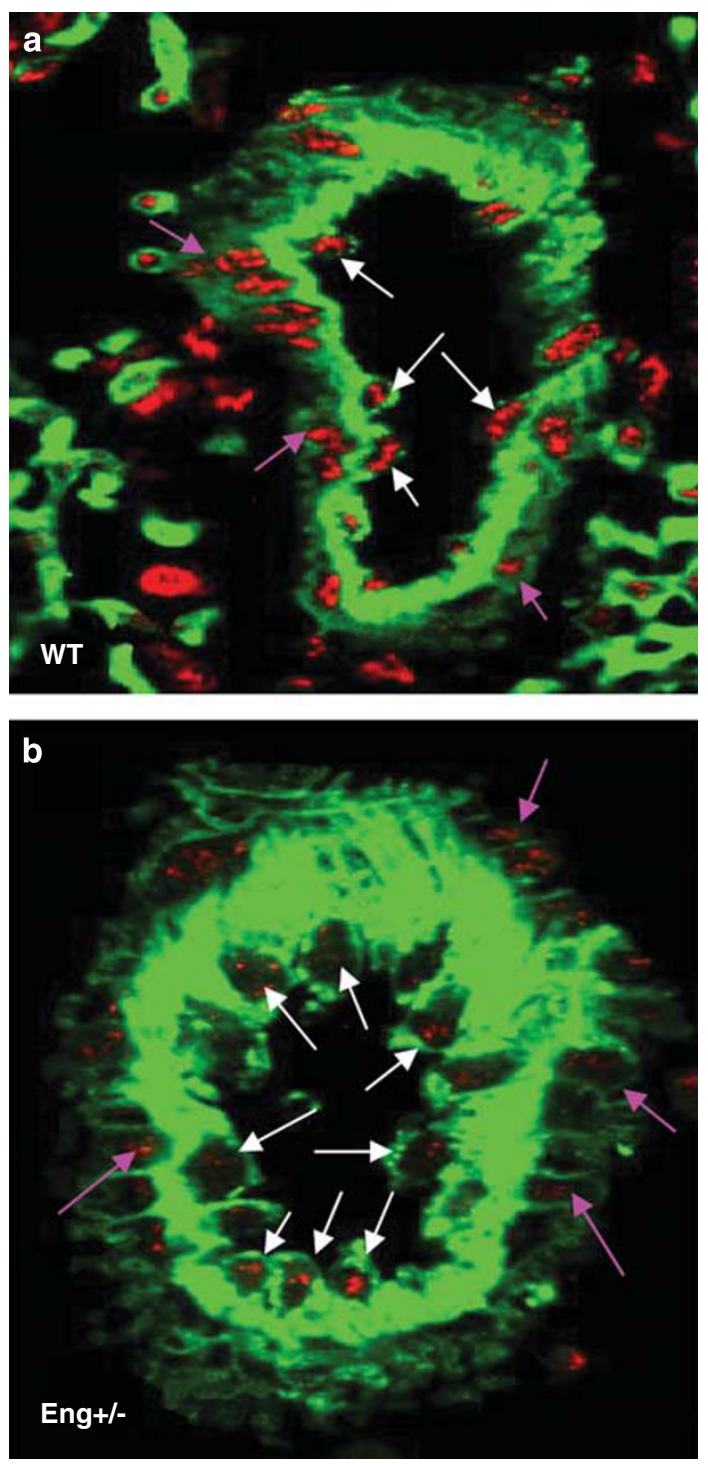

Figure 6 pSmad2 activity occurs in the majority of adult lung cells and appears to be reduced in Eng $+/-$ lungs. Double fluorescent staining of endothelial cells with isolectin-FITC (green ) and anti-pSmad2 antibody (red) shows extensive pSmad2 activity in the endothelial cells (white arrows) and neighbouring cells (purple arrows) of a distal artery in the wild-type lung (a). There appears to be reduced pSmad2 activity in some of the endothelial cells and all of the presumed smooth muscle cells in the Eng $+/$ - distal artery (b). A western blot of total lung protein extract also showed slightly reduced pSmad2 activity in Eng $+/$ - lung (c), but this difference was not found to be significant $(P=0.45)$, following densitometric analysis of western blots of total protein from lungs of four wild-type and four Eng $+/$ - mice (d).

comment definitively on expression in normal human lung. However, if the pattern of expression is similar in mouse and human, then reduced ENG or ACVRL1 levels in HHT or fPAH are most likely to affect the distal venules and arterioles, or capillaries of the lung. This would suggest that pulmonary AVMs are more likely to occur in the distal vasculature, and this would be consistent with a higher prevalence of PAVMs in the sub-pleural vasculature than in the deeper parts of the lung in human patients. ${ }^{20}$ Furthermore, the disproportionately short terminal/respiratory bronchioles in mouse compared with human, ${ }^{41}$ means that the distal arteries expressing Eng may also be dis-
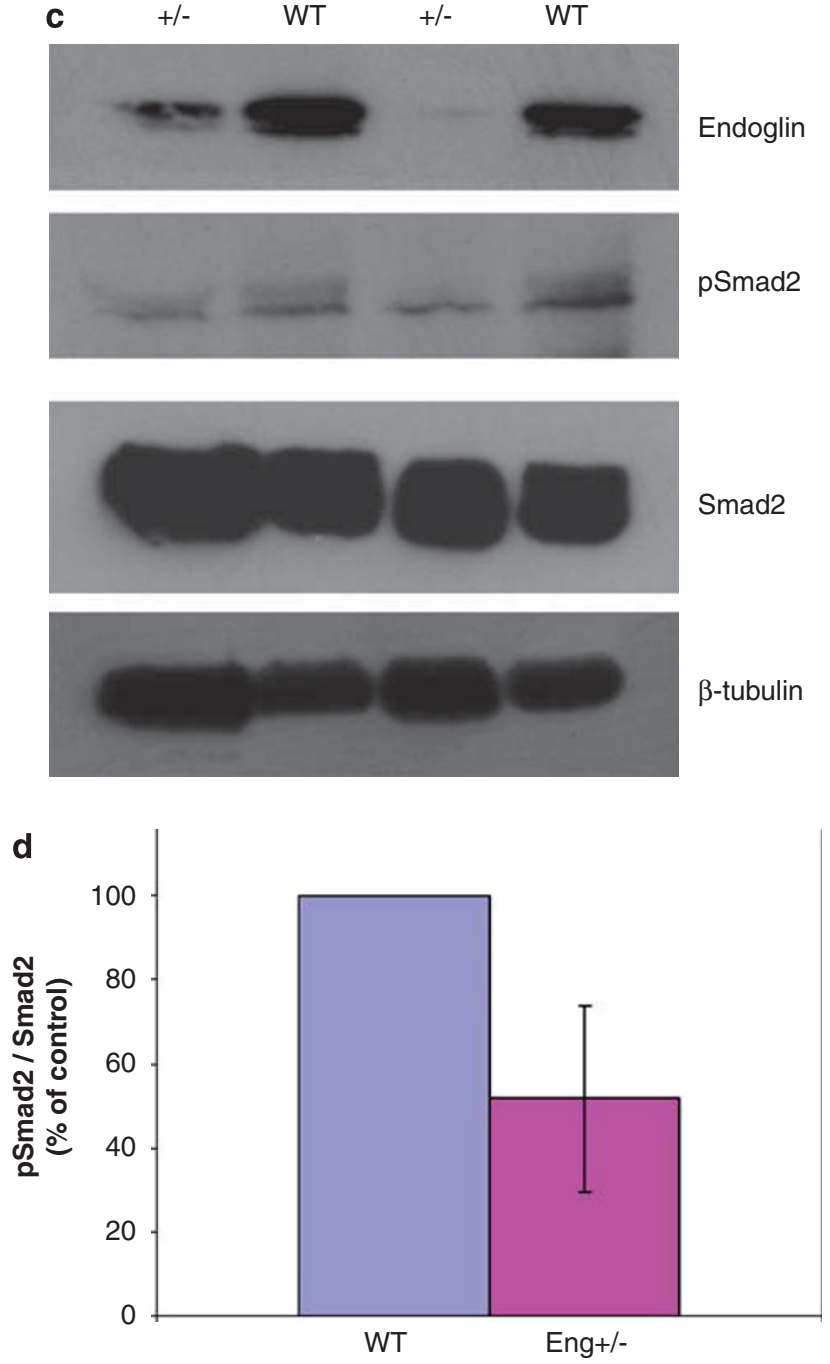

proportionately short in mouse and may be one reason why Eng $+/$ - mice do not appear to develop pulmonary AVMs.

Endoglin is expressed throughout veins in addition to distal vessels, a pattern which persists into adulthood and is not mirrored by Acvrll. Again, it is tempting to speculate that the broader expression of Eng than Acvrll in the pulmonary vasculature may explain why PAVMs are more frequent in HHT1 patients than in HHT2 patients. ${ }^{21}$ Co-expression of ACVRL1 and ENG in the distal arteries may be critical for regulating the endothelial cells of this region. We have shown that activation of $\mathrm{pSmad} 1 / 5 / 8$ in the endothelial cells of the distal arteries of adult lung is reduced 

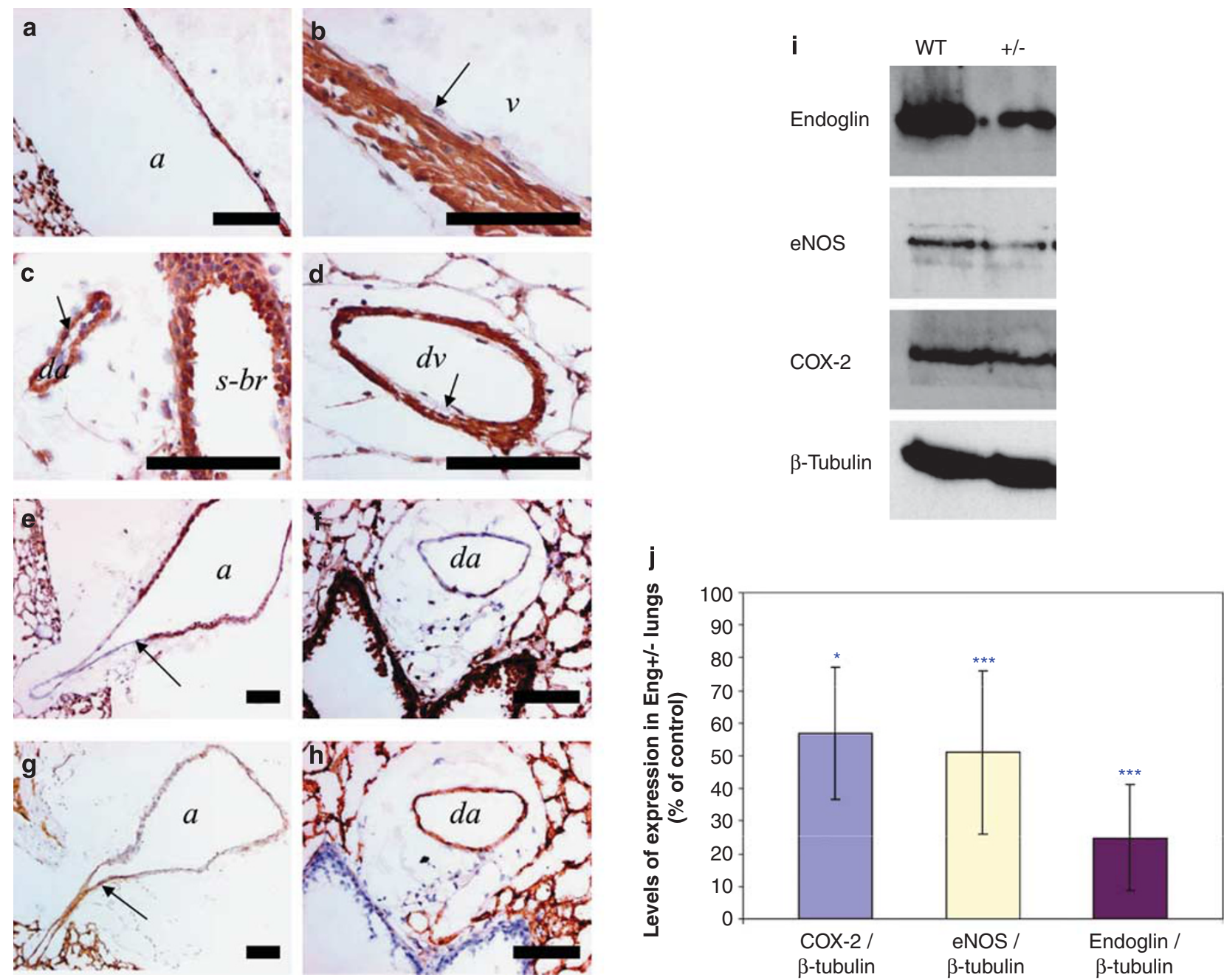

Figure 7 eNOS and Cox-2 expression in Eng $+/$ - lungs. Immunohistochemistry showing eNOS expression in endothelial cells of the proximal (a) and distal (c, arrow) pulmonary arteries, but not in proximal (b) or distal (d, arrow) pulmonary veins. Cox-2 is seen in the endothelial cells of the pulmonary arteries (e), but not the distal arteries (f) and has reciprocal expression to that of endoglin ( $\mathbf{g}, \mathbf{h})$. Note the reciprocal transition points for expression of Cox-2 (e, arrow) and endoglin (g, arrow). Western blots show modest reduction in both Cox-2 and eNOS levels in Eng $+/-$ mice (i), which was statistically significant following densitometric analysis of western blots of total protein from lungs of four wild-type and four Eng $+/-$ mice (j). (Cox-2, ${ }^{*} P=0.023$; eNOS, endoglin $\left.{ }^{* * *} P<0.001\right)$. For abbreviations see legend of Figure 1.

in Eng $+/$ - mice, suggesting that co-expression of Eng and Acvrll is important in promoting pSmad1/5/8 activation in vivo and correlates with previous in vitro data showing the importance of ENG in promoting activation of the BMP responsive Smads. ${ }^{34}$ Our results on the effect of reduced ENG signalling on pSmad2 activity are less clear-cut, partly because of the extensive $\mathrm{pSmad} 2$ activity throughout adult lungs. There appeared to be reduced pSmad2 activity in the smooth muscle cells surrounding distal arteries. This would be consistent with our previous findings, supporting the role of ENG in endothelial cells for downstream paracrine TGF- $\beta$ signalling to neighbouring smooth muscle cells. ${ }^{35}$ Interestingly, human vascular smooth muscle cells from the peripheral and proximal lung vessel explants have been shown to respond differently to BMP4 stimulation in vitro, ${ }^{42}$ and it is possible that this may reflect prior differential signalling from the endothelial cells to the underlying smooth muscle cells in line with the differential expression of Eng and Acvrll along the length of the arterial tree. Further work is required to investigate this possibility. Also, the exact nature of any common molecular pathology underlying HHT and fPAH that might explain the association of HHT genes with PAH disease remains to be determined. However, our work points to the co-expression of ACVRL1 and ENG, and associated pSmad1/5/8 signalling in the endothelial cells of distal arterioles as potentially important features. Another intriguing link was recently highlighted when the PAH symptoms in a paediatric ENG patient were relieved in later childhood as PAVMs developed. ${ }^{43,44}$ This is only one case, but illustrates the possibility that asymptomatic occluded pulmonary arterioles may be present in patients with PAVMs. 
It has been previously reported that Eng is primarily involved in regulating arterial tone and that expression of two key vasomediators, Cox-2 and eNOS, are altered in Eng + Imice. ${ }^{37,38,45}$ Our analysis of Eng expression in the lung indicates a direct role of Eng would be restricted to the distal pre-capillary arterioles. This is consistent with the architecture of the proximal conductance pulmonary arteries that are highly elastic and have little smooth muscle, whereas the vasoresponsive arteries are more distal. ${ }^{46}$ We did observe a small reduction in eNOS activity that was consistent in male mice, and agreed with previous reports. ${ }^{38,45}$ However, we did not see the previously reported increase in Cox- 2 activity and observed that in the arterial tree Cox-2 expression was reciprocal to that of Eng. In contrast to previous reports, we observed a modest decrease in Cox-2 expression in Eng + Ilungs. One possibility for this difference is the different methods used to solubilize proteins for fractionation on SDS gels. We aimed to solubilize total proteins in SDS sample buffer, while a previous report used a centrifugation step to pre-clear the lysate before solubilizing proteins. ${ }^{37}$ Other possible explanations may relate to the different mouse strains (NIH vs $\mathrm{C} 57 \mathrm{Bl} / 6$ ) and the different Eng mutations used in these analyses. ${ }^{30,47}$ It is also possible that previously reported differences in expression of Cox-2 and eNos in Eng $+1-$ mice are secondary responses that may sometimes be associated with HHT, but are not causal for HHT pathogenesis in the lung.

Taken together our data suggest a direct link between reduced Eng expression and lower levels of Smad1/5/8 activation in the vascular endothelium of distal pre-capillary arteries. It also points to the critical importance of this region in HHT, in addition to its known importance in PPAH. This study also serves to direct further work to define the cellular and molecular changes that lead to arteriovenous malformations in HHT to appropriate regions of the pulmonary vasculature.

\section{ACKNOWLEDGEMENTS}

We are grateful to Dr A Fisher for human lung tissue sections and to $R$ Hussain for genotyping. This work was supported by grants from the Wellcome Trust, British Heart Foundation, Cookson Trust and Borwick trust.

1. ten Dijke $P$, Arthur HM. Extracellular control of TGFbeta signalling in vascular development and disease. Nature Rev 2007;8:857-869.

2. Guttmacher $A E$, Marchuk DA, White Jr RI. Hereditary hemorrhagic telangiectasia. N Engl J Med 1995;333:918-924.

3. Shovlin $\mathrm{CL}$, Letarte $\mathrm{M}$. Hereditary haemorrhagic telangiectasia and pulmonary arteriovenous malformations: issues in clinical management and review of pathogenic mechanisms. Thorax 1999;54:714-729.

4. Pietra GG, Edwards WD, Kay JM, et al. Histopathology of primary pulmonary hypertension. A qualitative and quantitative study of pulmonary blood vessels from 58 patients in the National Heart, Lung, and Blood Institute, Primary Pulmonary Hypertension Registry. Circulation 1989:80:1198-1206.

5. Lane $\mathrm{KB}$, Machado RD, Pauciulo MW, et al. Heterozygous germline mutations in BMPR2, encoding a TGF-beta receptor, cause familial primary pulmonary hypertension. The International PPH Consortium. Nat Genet 2000;26:81-84.
6. Deng $\mathrm{Z}$, Morse $\mathrm{JH}$, Slager $\mathrm{SL}$, et al. Familial primary pulmonary hypertension (gene PPH1) is caused by mutations in the bone morphogenetic protein receptor-II gene. Am J Hum Genet 2000;67:737-744.

7. Harrison RE, Flanagan JA, Sankelo $M$, et al. Molecular and functional analysis identifies ALK-1 as the predominant cause of pulmonary hypertension related to hereditary haemorrhagic telangiectasia. J Med Genet 2003;40:865-871.

8. Bideau A, Plauchu $H$, Brunet $G$, et al. Epidemiological investigation of Rendu-Osler disease in France: its geographical distribution and prevalence. Population 1989;44:3-22.

9. Kjeldsen $A D$, Vase P, Green A. Hereditary haemorrhagic telangiectasia: a population-based study of prevalence and mortality in Danish patients. J Intern Med 1999;245:31-39.

10. Dakeishi M, Shioya T, Wada Y, et al. Genetic epidemiology of hereditary hemorrhagic telangiectasia in a local community in the northern part of Japan. Hum Mutat 2002;19:140-148.

11. McAllister KA, Grogg KM, Johnson DW, et al. Endoglin, a TGF-beta binding protein of endothelial cells, is the gene for hereditary haemorrhagic telangiectasia type 1. Nat Genet 1994;8:345-351.

12. Johnson DW, Berg JN, Baldwin MA, et al. Mutations in the activin receptor-like kinase 1 gene in hereditary haemorrhagic telangiectasia type 2. Nat Genet 1996;13:189-195.

13. Cole SG, Begbie ME, Wallace GM, et al. A new locus for hereditary haemorrhagic telangiectasia (HHT3) maps to chromosome 5. J Med Genet 2005;42:577-582.

14. Bayrak-Toydemir P, McDonald J, Akarsu N, et al. A fourth locus for hereditary hemorrhagic telangiectasia maps to chromosome 7. Am J Med Genet A 2006;140:2155-2162.

15. Gallione CJ, Repetto GM, Legius $E$, et al. A combined syndrome of juvenile polyposis and hereditary haemorrhagic telangiectasia associated with mutations in MADH4 (SMAD4). Lancet 2004;363: 852-859.

16. Barbara NP, Wrana JL, Letarte M. Endoglin is an accessory protein that interacts with the signaling receptor complex of multiple members of the transforming growth factor-beta superfamily. J Biol Chem 1999;274:584-594.

17. Scharpfenecker $M$, van Dinther $M$, Liu $Z$, et al. BMP-9 signals via ALK1 and inhibits bFGF-induced endothelial cell proliferation and VEGFstimulated angiogenesis. J Cell Sci 2007;120(Part 6):964-972.

18. David L, Mallet C, Mazerbourg S, et al. Identification of BMP9 and BMP10 as functional activators of the orphan activin receptor-like kinase 1 (ALK1) in endothelial cells. Blood 2007;109:1953-1961.

19. Braverman IM, Keh A, Jacobson BS. Ultrastructure and threedimensional organization of the telangiectases of hereditary hemorrhagic telangiectasia. J Invest Dermatol 1990;95:422-427.

20. Bosher Jr LH, Blake DA, Byrd BR. An analysis of the pathologic anatomy of pulmonary arteriovenous aneurysms with particular reference to the applicability of local excision. Surgery 1959;45:91-104.

21. Berg J, Porteous $M$, Reinhardt $D$, et al. Hereditary haemorrhagic telangiectasia: a questionnaire based study to delineate the different phenotypes caused by endoglin and ALK1 mutations. J Med Genet 2003;40:585-590.

22. Oh SP, Seki T, Goss KA, et al. Activin receptor-like kinase 1 modulates transforming growth factor-beta 1 signaling in the regulation of angiogenesis. Proc Natl Acad Sci USA 2000;97:2626-2631.

23. Seki T, Yun J, Oh SP. Arterial endothelium-specific activin receptor-like kinase 1 expression suggests its role in arterialization and vascular remodeling. Circ Res 2003;93:682-689.

24. Seki T, Hong KH, Oh SP. Nonoverlapping expression patterns of ALK1 and ALK5 reveal distinct roles of each receptor in vascular development. Lab Invest 2006;86:116-129.

25. Panchenko MP, Williams MC, Brody JS, et al. Type I receptor serinethreonine kinase preferentially expressed in pulmonary blood vessels. Am J Physiol 1996;270(4 Part 1):L547-L558.

26. Jonker L, Arthur HM. Endoglin expression in early development is associated with vasculogenesis and angiogenesis. Mech Dev 2002;110:193-196.

27. Torsney $E$, Charlton R, Parums D, et al. Inducible expression of human endoglin during inflammation and wound healing in vivo. Inflamm Res 2002;51:464-470.

28. Duff SE, Li C, Garland JM, et al. CD105 is important for angiogenesis: evidence and potential applications. FASEB J 2003;17:984-992. 
29. Alejandre-Alcazar MA, Michiels-Corsten M, Vicencio AG, et al. TGF-beta signaling is dynamically regulated during the alveolarization of rodent and human lungs. Dev Dyn 2008;237:259-269.

30. Arthur HM, Ure J, Smith AJ, et al. Endoglin, an ancillary TGFbeta receptor, is required for extraembryonic angiogenesis and plays a key role in heart development. Dev Biol 2000;217:42-53.

31. Millino C, Sarinella F, Tiveron C, et al. Cardiac and smooth muscle cel contribution to the formation of the murine pulmonary veins. Dev Dyn 2000;218:414-425.

32. Jones WK, Sanchez A, Robbins J. Murine pulmonary myocardium: developmental analysis of cardiac gene expression. Dev Dyn 1994;200:117-128.

33. Hislop A. Developmental biology of the pulmonary circulation. Paediatr Respir Rev 2005;6:35-43.

34. Lebrin F, Goumans MJ, Jonker L, et al. Endoglin promotes endothelia cell proliferation and TGF-beta/ALK1 signal transduction. EMBO J 2004;23:4018-4028.

35. Carvalho $\mathrm{RL}$, Jonker $\mathrm{L}$, Goumans $\mathrm{MJ}$, et al. Defective paracrine signalling by TGFbeta in yolk sac vasculature of endoglin mutant mice: a paradigm for hereditary haemorrhagic telangiectasia. Development 2004;131:6237-6247.

36. Quick CM, Hashimoto T, Young WL. Lack of flow regulation may explain the development of arteriovenous malformations. Neurol Res 2001;23:641-644.

37. Jerkic M, Rivas-Elena JV, Santibanez JF, et al. Endoglin regulates cyclooxygenase-2 expression and activity. Circ Res 2006;99: 248-256.
38. Toporsian M, Gros R, Kabir MG, et al. A role for endoglin in coupling eNOS activity and regulating vascular tone revealed in hereditary hemorrhagic telangiectasia. Circ Res 2005;96:684-692.

39. Fernandez LA, Garrido-Martin EM, Sanz-Rodriguez F, et al. Gene expression fingerprinting for human hereditary hemorrhagic telangiectasia. Hum Mol Genet 2007;16:1515-1533.

40. Feron $\mathrm{O}$, Belhassen L, Kobzik L, et al. Endothelial nitric oxide synthase targeting to caveolae. Specific interactions with caveolin isoforms in cardiac myocytes and endothelial cells. J Biol Chem 1996;271:22810-22814.

41. Bal HS, Ghoshal NG. Morphology of the terminal bronchiolar region of common laboratory mammals. Lab Anim 1988;22:76-82.

42. Yang $X$, Long $L$, Southwood $M$, et al. Dysfunctional Smad signaling contributes to abnormal smooth muscle cell proliferation in familial pulmonary arterial hypertension. Circ Res 2005;96:1053-1063.

43. Mache CJ, Gamillscheg A, Popper $\mathrm{HH}$, et al. Early-life pulmonary arterial hypertension with subsequent development of diffuse pulmonary arteriovenous malformations in hereditary haemorrhagic telangiectasia type 1. Thorax 2008;63:85-86.

44. Harrison RE, Berger R, Haworth SG, et al. Transforming growth factorbeta receptor mutations and pulmonary arterial hypertension in childhood. Circulation 2005;111:435-441.

45. Jerkic M, Rivas-Elena JV, Prieto $M$, et al. Endoglin regulates nitric oxidedependent vasodilatation. FASEB J 2004;18:609-611.

46. Dawson CA. Role of pulmonary vasomotion in physiology of the lung. Physiol Rev 1984;64:544-616.

47. Bourdeau A, Dumont DJ, Letarte M. A murine model of hereditary hemorrhagic telangiectasia. J Clin Invest 1999;104:1343-1351. 